\title{
Bubble babies and bathwater
}

Last month's rumors of the demise of gene therapy were greatly exaggerated. Despite headlines screaming "cancer scare," "halted trials," and "shock setback for gene cures"-not to mention talk of full-blown moratoriums on trials and going back to drawing boards - the disclosure of a serious adverse event in a three-yearold boy undergoing gene therapy for X-linked severe combined immune deficiency (SCID) did not represent a "death knell" for the field. For many gene therapies that do not involve integration into chromosomes, it was irrelevant. And for integrative gene therapies using retroviruses, it was not completely unexpected-in some respects, it is remarkable that this hasn't happened before.

The boy in question was taking part in a trial, led by Alain Fischer, Marina Cavazzana-Calvo, and their colleagues at the Necker Hospital in Paris, to find a cure for a type of SCID caused by a mutant form of the $\gamma \mathrm{c}$ interleukin receptor that blocks the development of T lymphocytes and natural killer cells. If left untreated or not placed in isolation (hence the term "bubble babies"), boys with this defect soon fall prey to even minor infections and normally die before reaching their first birthday.

In the French trial, stem cells are collected from the bone marrow, a common retroviral gene vector (murine leukemia virus) is used to deliver ex vivo the normal $\gamma c$ gene, and the recombinant cells are reintroduced into the patient's marrow by simple transfusion. Retrovirus is the vector of choice because it integrates foreign DNA into the chromosome (albeit randomly), potentially allowing stable and prolonged expression of the interleukin receptor over the patient's lifetime.

Until last spring, all patients enrolled in the trial underwent treatment without toxicity. Then, one patient (the three-year-old) suddenly developed a rapidly increasing $\gamma \delta \mathrm{T}$-cell count after a bout of chicken pox. By September, when the boy's count had climbed to 200,000 cells/ml, Fischer halted the trial, contacted the families of all the patients participating in the study, and informed centers carrying out similar trials in the United States, United Kingdom, and Germany of the adverse event. The affected boy was subsequently placed on chemotherapy for leukemia and is apparently now responding to this treatment.

Thus far, preliminary analysis of his blood indicates that the $\gamma_{c}$ gene landed inside $L M O-2$, an oncogene on chromosome 11. It thus seems possible that the retrovirus activated transcription of $L M O-2$ and that this, in combination with the chicken pox infection and any other number of predisposing genetic, epigenetic, and environmental factors, precipitated the aberrant proliferation of his $\mathrm{T}$ lymphocytes, leading to a leukemia-like illness.

This is the first direct clinical evidence of a link between integration of foreign DNA and cancer in humans. But is it such a surprise? The theoretical risk of mutagenesis due to insertion has been known for many years. Just this summer, a short study published in Science $(296,497,2002)$ indicated that retroviral gene insertion could trigger leukemia in mice. Indeed, although it was not clear whether the specific marker gene transduced in the study
( $d L N G F R$ ) itself contributed to the leukemia, German authorities regarded the paper's findings with sufficient seriousness to suspend all human trials using retroviruses.

But no therapies are without risks. And certainly, on the basis of present data, the benefits of SCID gene therapy clearly outweigh the risks. That is what the UK Gene Therapy Advisory Committee concluded last month. And that is what the US Recombinant DNA Advisory Committee (RAC) recommended to the Food and Drug Administration some days later. They were not wrong.

Since pioneering experiments in 1991 used retroviral gene therapy against adenosine deaminase deficiency, many millions of cells in many hundreds of individuals have been transduced with foreign genes; roughly half of these transfers have been accomplished using retroviruses. The adverse result in this X-linked SCID trial is the first documented case of mutagenesis leading to a malignant event.

And let us not forget that results to date from the French and UK trials of the SCID therapy have been nothing short of miraculous. Nine of 11 SCID boys treated with retroviral gene vectorsinfants who otherwise would have expected to live for a few months before succumbing to infection-are now enjoying a normal life with partially restored immune function. This level of therapeutic efficacy not only surpasses what one would expect from most small molecules; it finally lays to rest the myth that gene therapy can never work.

The key problem now is how to determine the likelihood that a retroviral vector will land in one of the 300 or so oncogenes in the genome and trigger cancer. We do not know yet whether the risk of insertional mutagenesis is one in a hundred or one in a million. We have not even started to identify the "hot spots" in the genome where a retrovirus might integrate.

To complicate matters further, the present system of documenting adverse events in patients enrolled in gene-therapy trials is woefully inadequate. In the US, for example, although the National Institutes of Health collect details on serious adverse events quarterly, this covers only incidents from trials funded by the NIHnearly all industry studies are excluded. As a result, it's difficult for a scientist (let alone a member of the general public) to learn details about the problems cropping up in gene-therapy studies.

Two years after the death of a patient in a University of Pennsylvania adenoviral gene-therapy trial hit the headlines, we still have no system for reporting financial alliances and conflicts of interest of trial sponsors. And a report published by the US Institute of Medicine last month (see p. 1071) highlights these inadequacies and the biases of the present system toward protecting institutions and companies rather than patients' rights.

Now is the time for changes to improve patient protections and restore public confidence in this technology. The first step toward doing that will be to create national databases of all trial data that will allow adverse events to be monitored and enrolled patients to be tracked systematically. 